\title{
Application of the amniotic fluid metabolome to the study of fetal malformations, using Down syndrome as a specific model
}

\author{
JUN HUANG ${ }^{1,2}$, JINHUA MO ${ }^{1,2}$, GUILI ZHAO ${ }^{1,2}$, QIYIN LIN ${ }^{1,2}$, GUANHUI WEI $^{2}$, \\ WEINAN DENG ${ }^{1,2}$, DUNJIN CHEN ${ }^{1,2}$ and BOLAN YU ${ }^{1,2}$ \\ ${ }^{1}$ Key Laboratory For Major Obstetric Diseases of Guangdong Province; ${ }^{2}$ Key Laboratory of \\ Reproduction and Genetics of Guangdong Higher Education Institutes, Third Affiliated Hospital \\ of Guangzhou Medical University, Guangzhou, Guangdong 510150, P.R. China
}

Received November 7, 2016; Accepted June 16, 2017

DOI: $10.3892 / \mathrm{mmr} .2017 .7507$

\begin{abstract}
Although monitoring and diagnosis of fetal diseases in utero remains a challenge, metabolomics may provide an additional tool to study the etiology and pathophysiology of fetal diseases at a functional level. In order to explore specific markers of fetal disease, metabolites were analyzed in two separate sets of experiments using amniotic fluid from fetuses with Down syndrome (DS) as a model. Both sets included 10-15 pairs of controls and cases, and amniotic fluid samples were processed separately; metabolomic fingerprinting was then conducted using UPLC-MS. Significantly altered metabolites involved in respective metabolic pathways were compared in the two experimental sets. In addition, significantly altered metabolic pathways were further compared with the genomic characters of the DS fetuses. The data suggested that metabolic profiles varied across different experiments, however alterations in the 4 metabolic pathways of the porphyrin metabolism, bile acid metabolism, hormone metabolism and amino acid metabolism, were validated for the two experimental sets. Significant changes in metabolites of coproporphyrin III, glycocholic acid, taurochenodeoxycholate, taurocholate, hydrocortisone, pregnenolone sulfate, L-histidine, L-arginine, L-glutamate and L-glutamine were further confirmed. Analysis of these metabolic alterations was linked to aberrant gene expression at chromosome 21 of the DS fetus. The decrease in coproporphyrin III in the DS fetus may portend abnormal erythropoiesis, and unbalanced glutamine-glutamate concentration was observed to be closely associated with abnormal brain development in the DS fetus. Therefore, alterations in amniotic fluid metabolites may provide
\end{abstract}

Correspondence to: Dr Bolan Yu, Key Laboratory for Major Obstetric Diseases of Guangdong Province, Room 806, Teaching Building, 63 Duobao Road, Guangzhou, Guangdong 510150, P.R. China

E-mail: yubolan-q@qq.com

Key words: embryo development, metabolomics, Down syndrome, amniotic fluid, biomarkers important clues to understanding the etiology of fetal disease and help to develop diagnostic testing for clinical applications.

\section{Introduction}

Birth defects and abnormal fetal growth constitute major fetal diseases associated with adverse pregnancy outcomes, including fetal structural anomalies, fetal chromosomal disorders, fetal growth retardation and abnormal fetal brain development or lung maturation (1). Although ultrasonographic methods, karyotyping, and developing noninvasive prenatal test techniques can assist in diagnosing most of the structural malformations and chromosomal disorders, the monitoring and diagnosis of other types of fetal developmental diseases in utero remain a challenge.

Amniotic fluid is the fluid within the amniotic sac that surrounds the fetus, providing physical protection and nutritional sources for the fetus $(2,3)$. Free diffusion occurs bi-directionally between the amniotic fluid and fetal skin, placenta and umbilical cord. Therefore, the composition of amniotic fluid is dynamic at different gestational ages, containing biomolecules from maternal plasma and fetal metabolism, in addition to cells originating from fetal kidney, heart, lung, liver and hematopoetic cell lineages (4). In the clinic, amniocentesis is routinely used to diagnose fetal chromosomal disorders such as Down syndrome (DS).

Previous studies suggest that amniotic fluid contains many biologically important molecules such as DNA, RNA and metabolites that may reflect fetal development and pregnancy condition, including preterm birth and preeclampsia $(2,5)$. For example, Larrabee et al (6) performed the first transcriptomic analysis of human amniotic fluid and observed that fetal gene expression was dynamic and changed over the course of gestation. Hui et al (7), using microarray analysis, identified that the transcriptome in mid-trimester amniotic fluid came from specific organs and physiological systems. Using proteomic profiling, Queloz et al (8) observed that amniotic fluid proteins were observed in differential abundances in early pregnancy compared with full term; and Romerao et al (9) analyzed preterm labor with and without intra-amniotic infection, and identified different biomarkers for these clinical subgroups comprising preterm labor. 
Metabolomics is a technique that encompasses individual metabolic profiles and their changes over time due to physiology, disease, toxicity or other effects (10). Due to the fact that in-vivo metabolites are the downstream products of gene expression and protein synthesis, they may more closely represent actual cellular activity at a functional level and at a specific point in time (10). At present, the most commonly used approaches for metabolic studies are liquid chromatography-coupled mass spectrometry (LC-MS) and nuclear magnetic resonance (NMR) spectroscopy, which can detect in-vivo metabolites, including amino acids, oligopeptides, sugars, steroids, biliary acids, fatty acids and other intermediary compounds (11). In 1994, Bock (12) profiled metabolites in the amniotic fluid by NMR to characterize second-trimester and third-trimester deliveries. Using ultra performance liquid chromatography and mass spectrometry (UPLC-MS), Graca et al (13) identified a decrease in specific amino acids and an increase in hexose in amniotic fluid samples taken from preterm deliveries. However, unlike genomic studies, variability exists in different research laboratories with respect to metabolomics analyses of fetal growth using amniotic fluid. The majority of this variability may be due to the short-term dynamics exhibited by metabolites, heterogeneous study populations, different metabolomic research approaches, and varied sampling and analysis protocols. Therefore, it remains a challenge to identify universal biomarkers such as $\alpha$-fetoprotein and human chorionic gonadotrophin for DS screening, which could be potentially applied for clinical use.

The aim of the current study was to explore whether stable and highly specific markers during different experiments in a relatively homogenous population carrying the same fetal disease. Fetal DS was selected as a model as it is one of the most common birth defects and is also a risk factor for fetal growth retardation. Due to the accuracy of DS diagnosis, this model would be more likely to consist of homogenous subjects and to represent characteristics of both fetal defects and growth retardation.

In the present study, metabolites were analyzed in two separate experiments that included a discovery set and a validation set, using amniotic fluid from DS fetuses. It was hypothesized that the metabolic alterations in amniotic fluid from DS fetuses cluster in specific metabolic pathways, and are associated with the altered gene expression observed at chromosome 21 during embryonic development of DS fetuses. To measure experimental repeatability, amniotic fluid samples were processed by different individuals separately, using the identical protocol, and metabolomic fingerprinting was conducted in these separate experiments using the same analytical approach. By analyzing the metabolic profiles of different experimental sets and comparing them to known genomic characteristics of DS fetuses, the present study aimed to validate them with consistency and repeatability regarding fetal disease, and to identify universal markers that would assist us in understanding the molecular mechanisms underlying fetal disease.

\section{Materials and methods}

Study population. The present study was approved by the Institutional Review Boards of the Third Affiliated Hospital of Guangzhou Medical University (Guangzhou, China) and 767 pregnant women with singleton pregnancies between
August 2014 and May 2015 each donated $5 \mathrm{ml}$ of amniotic fluid (AF), having provided written informed consent. The AF samples were collected from women undergoing amniocentesis for routine clinical indications (advanced maternal age, abnormal quadruple/triple test, family history of chromosomal abnormalities, suspected fetal anomalies or infection and upon maternal request) at the second trimester of gestation. The clinical outcomes from ultrasonogaphy, karyotyping and CMA results were additionally obtained. Pregnancies associated with an obstetric complication including hypertension, diabetes, premature rupture of the membranes or uterine infections were excluded from the AF analyses. Cases were a normal pregnancy with a DS fetus, and the controls were a normal pregnancy with a normal fetus, matched with the case samples in a 1:1 ratio based upon maternal age, fetal sex and gestational age. Finally, the discovery set consisted of 10 DS samples and 10 matched control samples. The validation set consisted of 15 DS samples and 15 matched controls.

AF sample collection. Transabdominal amniocentesis was performed with a 21-gauge needle under ultrasound guidance to evaluate the position of the fetus. A total of $5 \mathrm{ml}$ residual AF was collected for research purposes, in addition to that taken for diagnostic testing. Samples were transported immediately in a capped sterile syringe to the Biobank of the Third Affiliated Hospital of Guangzhou Medical University. The AF samples were then centrifuged for $10 \mathrm{~min}$ at $500 \mathrm{x} \mathrm{g}$ at $4^{\circ} \mathrm{C}$, and the supernatant and pellet cells were separately stored in aliquots at $-80^{\circ} \mathrm{C}$. The processing time was $<4 \mathrm{~h}$ from the time of sample collection to the time of sample freezing.

Chemicals and columns. LC-MS grade organic solvents, including acetonitrile, methanol, ammonium acetate, ammonium fluoride, ammonia, and formic acid, were purchased from Sigma (Sigma-Aldrich; Merck Millipore, Darmstadt, Germany). The HILIC column was Waters, ACQUITY UPLC BEH Amide $1.7 \mu \mathrm{m}, 2.1 \times 100 \mathrm{~mm}$ column; and the HSS T3 column was ACQUITY UPLC HSS T3 $1.8 \mu \mathrm{m}, 2.1 \times 100 \mathrm{~mm}$ column (Waters US, Milford, MA, USA).

Sample preparation. For proteins precipitation, $100 \mu \mathrm{l}$ of AF sample was thawed at $4^{\circ} \mathrm{C}$, and $400 \mu \mathrm{l}$ of cold $\left(-20^{\circ} \mathrm{C}\right)$ mixture of methanol/acetonitril $(1: 1, \mathrm{v} / \mathrm{v})$ was added. Samples were vortex-mixed and stored at $-20^{\circ} \mathrm{C}$ for $10 \mathrm{~min}$. Following centrifugation at $14,000 \mathrm{xg}$ for $15 \mathrm{~min}$ at $4^{\circ} \mathrm{C}$, the supernatant was filtered using a $0.22 \mu \mathrm{m}$ nylon filter and dried under vacuum. For LC-MS analysis, $100 \mu 1$ acetonitrile/water (1:1, $\mathrm{v} / \mathrm{v}$ ) was added to each sample, vortexed and centrifuged at $14,000 \mathrm{x} \mathrm{g}$ for $15 \mathrm{~min}$ at $4^{\circ} \mathrm{C}$ for final injection. QC samples were prepared by pooling equal volumes of each sample.

LC-QTOF-MS analysis. The UPLC system (Agilent 1290 Infinity LC System; Agilent Technologies, Inc., Santa Clara, CA, USA) consisting of a degasser, two binary pumps and a thermostated autosampler (maintained at $4^{\circ} \mathrm{C}$ ) coupled with a Triple TOF 5600 mass spectrometer (AB SCIEX, Framingham, MA, USA) were used for metabolomic analysis. Briefly, a $4.0 \mu 1$ sample of extracted AF samples was injected into a thermostated $\left(25^{\circ} \mathrm{C}\right)$ reverse-phase ACQUITY UPLC HSS T3 C18 column or ACQUITY UPLC BEH Amide 
column. The flow rate was $600 \mu \mathrm{l} / \mathrm{min}$ with solvent $\mathrm{A}$ (water with $0.1 \%$ formic acid) and solvent B (acetonitrile with $0.1 \%$ formic acid). QC samples were prepared by pooling equal volumes of each sample and injected at the beginning and at the end of each analysis, and at every five samples, to provide a measurement of the system's stability and performance in addition to reproducibility of the sample treatment procedure.

For the HILIC column, solvent A was composed of water and $25 \mathrm{mM}$ ammonium acetate and $25 \mathrm{mM}$ ammonia, and solvent B was acetonitrile. The chromatographic gradient started at $85 \%$ for phase B for the first minute, decreased to $65 \%$ from $1-12 \mathrm{~min}$, and then to $40 \%$ from $12-12.1 \mathrm{~min}$. The phase remained at $40 \%$ for solvent B from 12.1 to $15 \mathrm{~min}$, returned to $85 \%$ for phase $\mathrm{B}$ from $15-15.1 \mathrm{~min}$, and remained at $85 \%$ for phase $\mathrm{B}$ for $5 \mathrm{~min}$.

For the positive ion mode of the HSS T3 column, solvent A was composed of $0.1 \%$ formic acid and water, and solvent $\mathrm{B}$ was $0.1 \%$ formic acid and acetonitrile. For the negative ion mode of the HSS T3 column, solvent A was comprised of $0.5 \mathrm{mM}$ ammonium fluoride, and solvent B was acetonitrile. The chromatographic gradient started at $1 \%$ for phase $\mathrm{B}$ for the first $1.5 \mathrm{~min}$, and increased to $99 \%$ from $1.5-13 \mathrm{~min}$. The phase remained at $99 \%$ for solvent $\mathrm{B}$ from $13-16.5 \mathrm{~min}$, returned to $1 \%$ for phase $\mathrm{B}$ from $16.5-16.6 \mathrm{~min}$, and remained at $1 \%$ phase for B for $4 \mathrm{~min}$.

The samples were separated by UPLC and analyzed with a Triple TOF 5600 mass spectrometer, using electrospray ionization (ESI) for positive ion and negative ion modes, respectively. For the HILIC column, the ESI source conditions were ion source gas 1 (Gas 1), 60; ion source gas 2 (Gas 2), 60; curtain gas (CUR), 20; source temperature, $600^{\circ} \mathrm{C}$; and ionspray voltage floating (ISVF) $\pm 5,500 \mathrm{~V}$ (positive and negative modes). The detector conditions were TOF MS scan m/z range, 50-1,000 Da; product ion scan $\mathrm{m} / \mathrm{z}$ range, 25-1,000 Da; scan accumulation time, $0.20 \mathrm{~s} / \mathrm{spectra}$; product ion scan accumulation time, $0.05 \mathrm{~s} / \mathrm{spectra}$; declustering potential (DP), $\pm 60 \mathrm{~V}$ (positive and negative modes); and collision energy, $35 \pm 15 \mathrm{eV}$. MS/MS was acquired using information-dependent acquisition (IDA) and high-sensitivity mode; the IDA set excluded isotopes within $4 \mathrm{Da}$, and 6 candidate ions were monitored per cycle.

For the HSS T3 column, the ESI source conditions were Gas 1, 40; Gas 2, 80; CUR, 30; source temperature, $650^{\circ} \mathrm{C}$; and the ISVF $\pm 5,000 \mathrm{~V}$ (positive and negative ion modes). The detector conditions were TOF MS scan m/z range, 60-1,000 Da; product ion scan $\mathrm{m} / \mathrm{z}$ range, 25-1,000 Da; TOF MS scan accumulation time, $0.20 \mathrm{~s} / \mathrm{spectra}$; product ion scan accumulation time, $0.05 \mathrm{~s} /$ spectra; DP, $\pm 60 \mathrm{~V}$ (positive and negative modes); and collision energy, $35 \pm 15 \mathrm{eV}$. MS/MS was acquired using IDA with high-sensitivity mode; the IDA set excluded isotopes within $4 \mathrm{Da}$, and 6 candidate ions were monitored per cycle.

Bioinformatics analysis. Kyoto Encyclopedia of Genes and Genomes (KEGG) pathway analysis (http://www.genome .jp/kegg/) was performed with Database for Annotation, Visualization and Integrated Discoverybioinformatics website (https://david.ncifcrf.gov/) platform using default parameters. Gene classes were identified based on Gene Ontology term categories and the KEGG pathway database.
Compound identification and statistical analyses. Raw data acquired using the UHPLC-MS system was processed to mzML format via ProteoWizard software version 3.0.6458 (http://proteowizard.sourceforge.net) for subsequent data analysis (14). The background cleaning and alignment of drift (by retention time and mass), and filtering of the data collected by LC-MS were performed with XCMS (http://metlin.scripps .edu/download) (15). Each compound was described by mass, retention time and abundance. Identification of the compounds was confirmed by accurate mass and MS/MS profiles, and confirmation was performed with standards by comparison of retention time, isotopic distribution and fragments of commercially available reagents with those obtained from analyzed samples. For univariate statistical analysis, data was uniformed using autoscaling and multi-dimensional analysis, including PCA and PLS-DA, using Metabo Analysis software version 3.0 (www.metaboanalyst.ca) (16). Numerical data are shown as mean \pm standard deviation, and independent samples Student's t-test and volcano analysis were performed using the $\mathrm{R}$ package.

\section{Results}

Patient characteristics. A total of 25 cases of fetal DS and 25 controls were recruited in the current study, in 2 separate sets. In the discovery set, the average maternal age of cases and controls was 32 years, and the average gestational ages at amniocentesis were 131 and 130 days, respectively (Table I). In the validation set, the average maternal ages of cases and controls were 34 and 33 years, respectively; and the average gestational age at amniocentesis was 131 days (Table I). The fetal sex rate was identical in cases and the control group in both sets (Table I).

Metabolomic fingerprinting. The UPLC-Q-TOF-MS system was applied to AF fingerprinting. In total, 20 samples of the discovery set and 30 samples of the validation set were analyzed in 2 separate experiments. Unsupervised principal component analysis (PCA-X) models (Fig. 1) demonstrated good clustering of all QC samples in all HILLC(+), HILLC(-), HSS(+), and HSS(-) modes in the 2 experimental sets, which indicated that the system was stable and performance was reliable. The detected peak numbers were different for the two sets: In the discovery set, the peak numbers in HILIC(+), HILIC(-), HSS(+), and HSS(-) modes were 4,044, 3,880, 5,877 and 3,970, respectively; while in the validation set, the peak numbers in HILIC(+), HILIC(-), HSS(+), and HSS(-) modes were $5,915,6,029,4,801$ and 5,183 , respectively.

In the unsupervised PCA-X analysis, 5 outliers were observed in the discovery set, and 4 of them were from controls and 1 from cases. Similarly, 8 outliers were observed in the discovery set, and 3 of them were from controls, and 5 from cases. Therefore, in later analysis, these outliers were eliminated.

Supervised partial least-squares discriminate analysis (PLS-DA) was further used to model the differences between cases and controls. The PLS-DA model demonstrated clear separation of samples from the 2 groups (Fig. 2). In the discovery set, the explained variance (R2) and predicted variance (Q2) in $\operatorname{HILIC}(+), \operatorname{HILIC}(-), \operatorname{HSS}(+)$, and $\operatorname{HSS}(-)$ 
Table I. Demographic characteristics of study subjects.

\begin{tabular}{lccccc}
\hline & \multicolumn{2}{c}{ Discovery set } & & \multicolumn{2}{c}{ Validation set } \\
\cline { 2 - 3 } \cline { 5 - 5 } Subject characteristics & Down syndrome & Control & & Down syndrome & Control \\
\hline Number of samples & 10 & 10 & & 15 & 15 \\
Maternal ages, years & $32(43-22)$ & $32(39-24)$ & & $34(41-23)$ & $33(39-21)$ \\
Gestational ages, days & $131(143-113)$ & $130(143-112)$ & & $131(164-112)$ & $131(163-112)$ \\
Fetal sex & 6 female & 6 female & & 6 female & 6 female \\
& 4 male & 4 male & & 9 male & 9 male \\
\hline
\end{tabular}

modes were $\mathrm{R} 2=1.00, \mathrm{Q} 2=0.31, \mathrm{R} 2=1.00, \mathrm{Q} 2=0.58, \mathrm{R} 2=1.00$, $\mathrm{Q} 2=0.36, \mathrm{R} 2=0.96$ and $\mathrm{Q} 2=0.74$, respectively. In the validation set, the R2 and Q2 in HILIC(+), HILIC(-), HSS(+), and HSS(-) modes were $\mathrm{R} 2=0.99, \mathrm{Q} 2=0.35, \mathrm{R} 2=0.98, \mathrm{Q} 2=0.31, \mathrm{R} 2=0.98$, $\mathrm{Q} 2=0.65, \mathrm{R} 2=0.98$ and $\mathrm{Q} 2=0.69$, respectively. Therefore, the HSS(-) mode was the best mode for PLS-DA analysis in both the discovery and validation sets.

Metabolite analysis. Peaks that were significantly different in the 4 modes of the 2 experimental sets were initially screened, and some of them had been identified and validated using standards. Those identified in both the discovery set and validation set, and that had a similar change in tendency and in significance $(\mathrm{P}<0.05$ or $\mathrm{P}<0.1$ for both experiments) were chosen as the final identified markers (Table II). These were coproporphyrin-III, pregnenolone sulfate, taurochenodeoxycholate, L-arginine, taurocholate, hydrocortisone (cortisol), L-histidine, glycocholic acid, L-glutamate and L-glutamine. Among these markers, amounts of hydrocortisone and L-glutamine were increased significantly, however the quantities of the other metabolites were decreased in cases when compared with the controls. When the quantities were assessed in individual samples for both the discovery set and the validation set, 10 metabolites demonstrated a significant difference in cases vs. controls (Fig. 3). The amounts of coproporphyrin-III, L-glutamate, pregnenolone sulfate, taurochenodeoxycholate, L-arginine and taurocholate were significantly decreased in cases compared with the controls $(\mathrm{P}<0.01)$, as were L-histidine and glycocholic acid $(\mathrm{P}<0.05)$. The levels of hydrocortisone and L-glutamine were significantly increased in cases compared with controls $(\mathrm{P}<0.01$ and $\mathrm{P}<0.05$, respectively).

KEGG analysis. Using KEGG pathway analysis, it was identified that there were at least 30 KEGG pathways altered in cases compared with the controls (Table III), including biosynthesis of amino acids, ABC transporters, alanine, aspartate and glutamate metabolism, bile secretion, arginine and proline metabolism, histidine metabolism, taurine and hypotaurine metabolism. The majority of these molecules are involved largely with amino acid metabolism, liver function, growth hormone and neural development. Notably, using KEGG pathways, certain molecules were shared with genes on chromosome 21. There were 9 KEGG pathways altered in AF from DS fetuses associated with genes on chromosome 21 (Table IV). These were galactose metabolism, purine metabolism, histidine metabolism, $\mathrm{ABC}$ transporters, neuroactive ligand-receptor interaction, Parkinson's disease, ALS, Huntington's disease and pathways in cancer.

\section{Discussion}

Although metabolomics has been applied to numerous biomedical studies, few have been conducted using this approach for the monitoring of fetal malformations. One of the reasons for this is the variability that exists in metabolomic studies in different experimental paradigms and laboratories. For example, using 1H-NMR, Graca et al $(13,17,18)$ identified alterations in metabolites including methionine and succinate with fetal malformations, however using UPLC-MS, they identified another group of metabolites that were not identical to those previously identified. In addition, Bock (12) studied the AF metabolome in fetal DS specimens, however the metabolites exhibited no particular pattern due to the small number collected. These researchers indicated that the results of a metabolomics study may largely depend on the study population, sampling process and detection approach when applied to the study of fetal disease.

To explore the repeatability and stability of AF metabolite identification, the discovery and validation sets were carefully designed, both of which included 10-15 pairs of controls and cases of DS fetuses (Table I). The results indicated that although $\sim 80$ and $\sim 60$ metabolites, respectively, were indentified in the two sets (which were significantly different between cases and controls), there were few metabolites that were common between the sets. The results demonstrated that metabolite identification may exhibit large variability among different samples, even when using the same approach and protocol. This is suggested to be due to the dynamic nature and inherent variability of metabolites in AF, and the limited number of techniques available to accurately identify them. However, when metabolomic pathways were investigated, the majority of the altered metabolites identified could be clustered into similar pathways in the two experiments (Table II). The major pathway alterations in DS fetuses included amino acids, bile secretion, neuroactive ligand-receptor interaction and galactose metabolism, all of which could be associated with the pathophysiology of DS fetuses, including growth and mental retardation (Table II). Therefore, although metabolite identification is not consistent in $\mathrm{AF}$, the alterations in metabolism pathways may be more likely to be invariable over different experiments. 

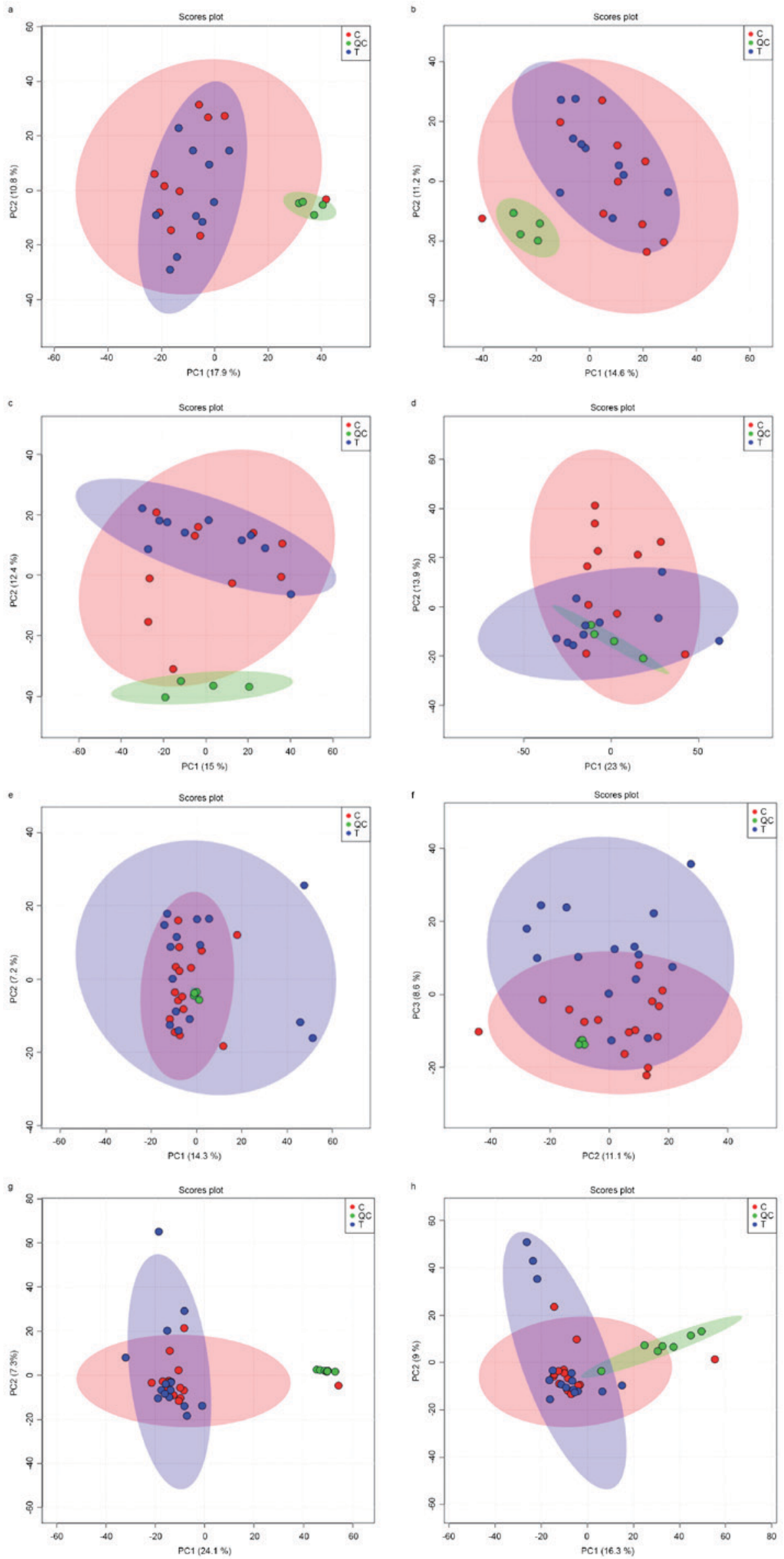

Figure 1. PCA-X score plots for samples and QC samples in the discovery set and validation set. (A-D) Discovery set. (E-H) Validation set. Blue circles, cases; red circles, controls; green circles, QC samples. (A and E) HILLC(+) mode; (B and F) HILLC(-) mode; (C and G) HSS(+) mode; (D and H) HSS(-) mode. PCA-X, principal component analysis; QC, quality control; HILLC, hydrophilic interaction chromatography; HSS, silica-based bonded phase. 

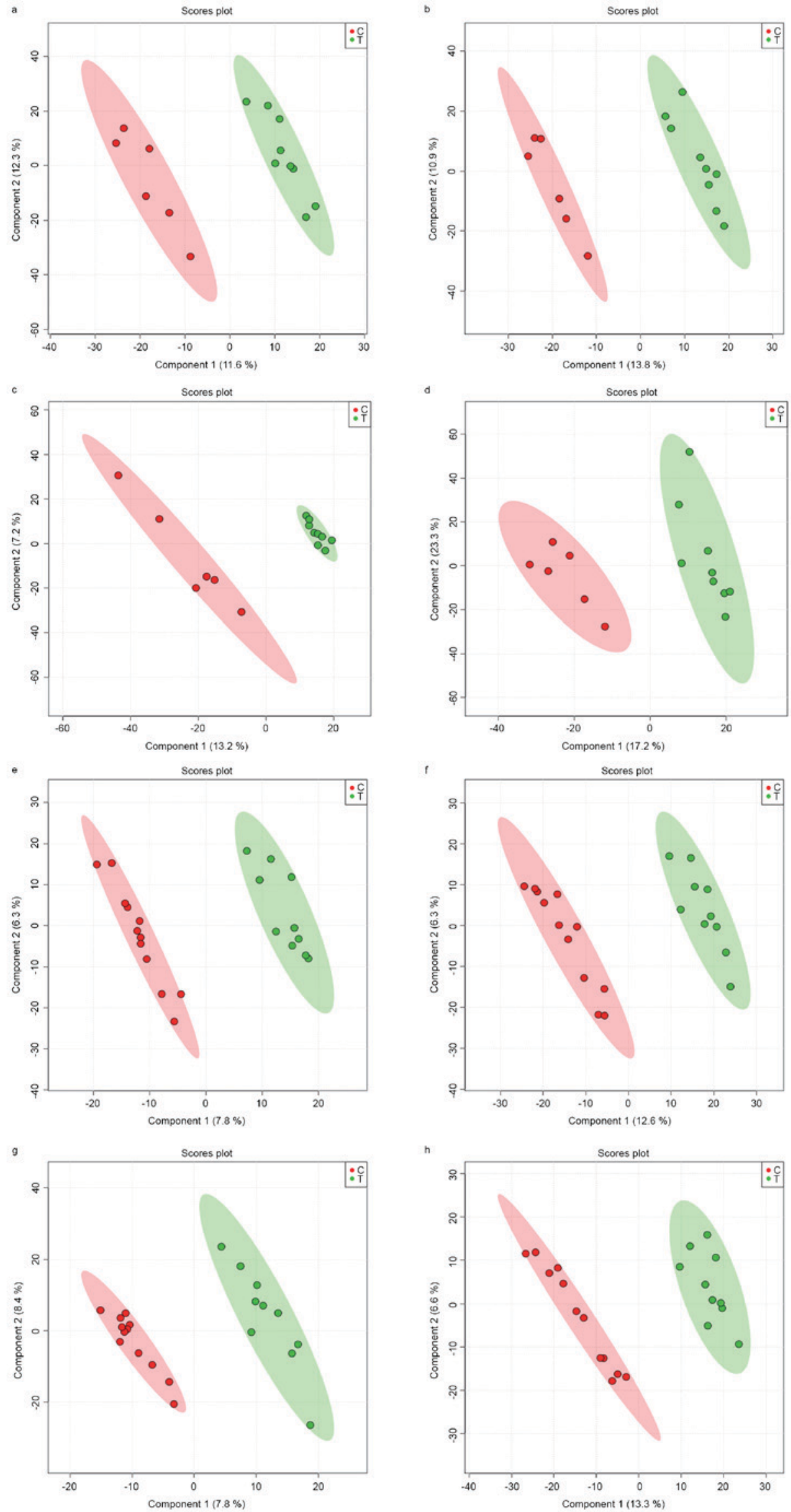

Figure 2. Supervised PLS-DA model score in discovery set and validation set. Green circles, cases; red circles, controls. (A-D) represent the data of discovery set obtained by PC1 vs. PC2 in HILLC(+), HILLC(-), HSS(+) and HSS(-) mode. The R2 and Q2 in (A-D) are R2=1.00, Q2=0.31, R2=1.00, Q2=0.58, R2=1.00, $\mathrm{Q} 2=0.36, \mathrm{R} 2=0.96$ and $\mathrm{Q} 2=0.74$, respectively. $(\mathrm{E}-\mathrm{H})$ represent the data of validation set obtained by PC1 vs. PC2 in HILLC(+), HILLC(-), HSS(+) and HSS(-) mode. The R2 and Q2 in (A-D) plots are R2 $=0.99, \mathrm{Q} 2=0.35, \mathrm{R} 2=0.98, \mathrm{Q} 2=0.31, \mathrm{R} 2=0.98, \mathrm{Q} 2=0.65, \mathrm{R} 2=0.98$ and $\mathrm{Q} 2=0.69$, respectively. PLS-DA, supervised partial least-squares discriminate analysis; HILLC, hydrophilic interaction chromatography; HSS, silica-based bonded phase. 
Table II. Altered KEGG pathways and involved metabolite numbers in two sets.

\begin{tabular}{|c|c|c|}
\hline Name & Discovery & Validation \\
\hline Metabolic pathways & 26 & 15 \\
\hline Biosynthesis of amino acids & 9 & 4 \\
\hline Biosynthesis of antibiotics & 7 & 4 \\
\hline Bile secretion & 6 & 4 \\
\hline Tryptophan metabolism & 5 & 3 \\
\hline Two-component system & 4 & 2 \\
\hline Purine metabolism & 2 & 2 \\
\hline $\mathrm{ABC}$ transporters & 8 & 5 \\
\hline Biosynthesis of secondary metabolites & 14 & 4 \\
\hline Central carbon metabolism in cancer & 10 & 4 \\
\hline Protein digestion and absorption & 9 & 4 \\
\hline Aminoacyl-tRNA biosynthesis & 8 & 4 \\
\hline $\begin{array}{l}\text { Biosynthesis of alkaloids derived } \\
\text { from ornithine, lysine and nicotinic acid }\end{array}$ & 7 & 2 \\
\hline $\begin{array}{l}\text { Microbial metabolism in diverse } \\
\text { environments }\end{array}$ & 5 & 4 \\
\hline $\begin{array}{l}\text { Alanine, aspartate and glutamate } \\
\text { metabolism }\end{array}$ & 4 & 2 \\
\hline Glyoxylate and dicarboxylate metabolism & $\mathrm{m} 4$ & 2 \\
\hline Alcoholism & 3 & 2 \\
\hline Primary bile acid biosynthesis & 3 & 3 \\
\hline GABAergic synapse & 2 & 2 \\
\hline Galactose metabolism & 2 & 2 \\
\hline Glutamatergic synapse & 2 & 2 \\
\hline Histidine metabolism & 2 & 3 \\
\hline Glutathione metabolism & 2 & 2 \\
\hline Nitrogen metabolism & 2 & 2 \\
\hline $\begin{array}{l}\text { Porphyrin and chlorophyll } \\
\text { metabolism }\end{array}$ & 3 & 2 \\
\hline $\begin{array}{l}\text { Neuroactive ligand-receptor } \\
\text { interaction }\end{array}$ & 3 & 3 \\
\hline Secondary bile acid biosynthesis & 3 & 3 \\
\hline Arginine and proline metabolism & 3 & 4 \\
\hline $\begin{array}{l}\text { D-Glutamine and D-glutamate } \\
\text { metabolism }\end{array}$ & 2 & 2 \\
\hline $\begin{array}{l}\text { Proximal tubule bicarbonate } \\
\text { reclamation }\end{array}$ & 4 & 2 \\
\hline Amyotrophic lateral sclerosis & 2 & 2 \\
\hline $\begin{array}{l}\text { Taurine and hypotaurine } \\
\text { metabolism }\end{array}$ & 2 & 2 \\
\hline
\end{tabular}

When the significantly altered metabolites were identified from both experiments, it was noted that they belonged to 4 categories: 4 were amino acids, 3 were bile acids, 2 were hormones and 1 was porphyrin (Table II, Fig. 3). Alterations in these metabolites have been associated with several negative pregnancy outcomes including abortion, fetal growth abnormality, prematurity and low birth weight (19). For example, in a rat intrauterine growth restriction model, elevated maternal and fetal corticosterone levels were reported in serum and AF (20). In humans, analysis of total urinary steroids was effective in detecting fetuses with Smith-Lemli-Opotz syndrome (21), and progesterone levels were altered in $87 \%$ of maternal urine in the presence of a DS fetus (22). Concomitantly, increased levels of cortisol and decreased levels of pregnenolone sulfate were detected in DS fetuses, which may be associated with abnormal bone and brain development. Similarly, bile acids including taurochenodeoxycholic acid, glycocholic acid and taurocholic acid all increased in AF from women with preterm deliveries (23). Due to the fact that the accumulation of bile acids can trigger an inflammatory response in maternal and fetal lungs (24), high circulating levels in the preterm birth fetus may reflect uterine stress due to preterm birth. Considering that mothers who carry DS fetuses did not exhibit a higher incidence of pregnancy complications, the decrease in bile acids that was observed may reflect an underdeveloped liver or impaired bile acid metabolism in the DS fetus.

In addition to changes in hormones and bile salts, altered amino acid concentrations are widely reported for fetuses in adverse pregnancies $(23,25)$. A previous study demonstrated that amino acids can be actively taken up by the fetus, and that metabolic factors such as insulin-like growth factor-1 treatment may promote gut utilization of amino acids from the AF pool (26). Among amino acids, arginine is the donor for NO in vivo, and its concentration is associated with decreased NO levels. Molecular mechanistic studies suggest that arginine is involved in diverse functions including placental angiogenesis, antioxidant stress, the immune system and placental apoptosis (27-30). In the present study, the decrease in L-arginine concentration suggested that the impaired growth of DS fetuses may also be involved in downregulating the NO signaling pathway, however the details warrant further validation. As an essential amino acid for fetuses, a decrease in histidine levels has been reported in several types of abnormal fetal growth including preterm fetuses and fetuses with skeletal dysplasia $(23,25)$. However, in the case of chorioamnionitis, histidine increased significantly, suggesting an association with uterine inflammation and infection (31). The majority of cases of DS fetuses are not accompanied by uterine infection, and therefore, similar to the situation for preterm fetuses and fetuses with skeletal dysplasia, the reduction in histidine in AF may be due to altered histidine metabolism.

As a relatively recent type of 'omics' technology, one of the most important goals of metabolomics is to assist in uncovering the etiology of diseases at functional levels. It is notable that in the present study, although only certain significantly altered metabolites were identified, some were directly associated with the DS phenotype at a molecular level. It was identified that metabolites involved in erythropoiesis (coproporphyrin III) were significantly altered in the DS fetus. Coproporphyrin III is a byproduct of heme biosynthesis, and is excreted normally in feces as a decomposition product of bilirubin; it has been demonstrated that coproporphyrin increases in human urine due to lead poisoning, which is associated with decomposition products of erythropoiesis (32). A significant decrease in coproporphyrin III was observed in AF taken from fetuses with DS, which may reflect an aberration in fetal erythropoiesis. Correspondingly, patients with DS frequently exhibit blood cell abnormalities, including abnormal blood counts, transient myeloproliferative disorders and acute megakaryoblastic leukemia. Using a stem cell model, DS cells 

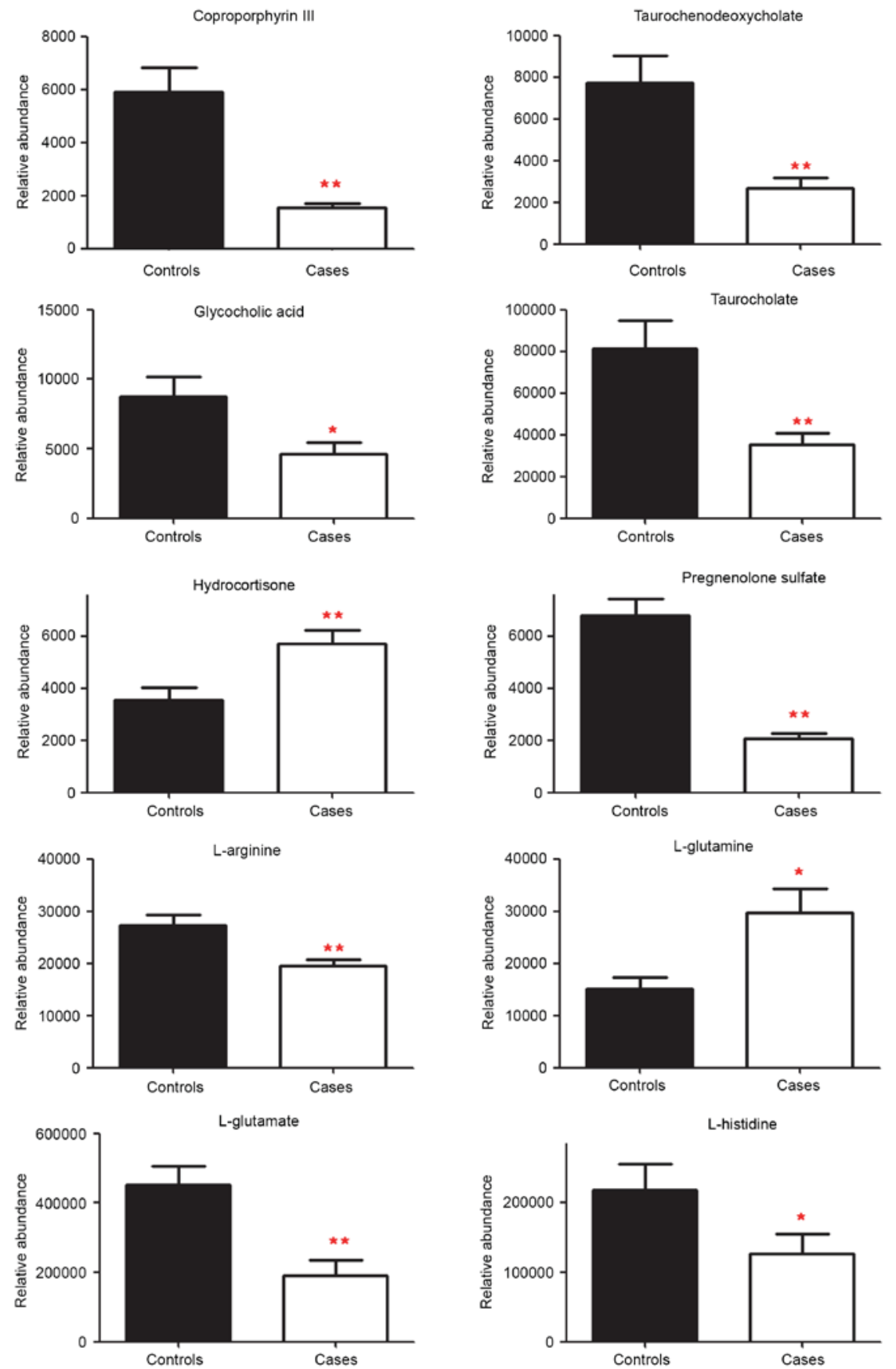

Figure 3. Significant changes of 10 markers in both discovery set and validation set. Figures indicate average values of relative abundance and significance between cases and controls. ${ }^{*} \mathrm{P}<0.05,{ }^{* *} \mathrm{P}<0.01$.

were demonstrated to exhibit enhanced erythropoiesis and reduced myelopoiesis (33). In vitro and mouse transplantation assays have indicated that trisomy 21 progenitors manifested enhanced production of erythroid and megakaryocytic cells that proliferated excessively (34). Therefore, the decrease in coproporphyrin III in DS fetuses may be potentially coupled at a molecular level to their abnormal erythropoiesis.

There have been few metabolomics studies of fetal malformations that make intrinsic associations between metabolites and fetal disease genotypes, likely due to the fact that the majority of experiments used a mixture of fetal abnormality samples that included abnormalities in the central nervous, cardiac and urogenital systems $(5,35)$. Therefore, whether the significantly altered metabolites could directly reflect the etiology of fetal diseases or whether they are by-products of affected signaling pathways in malformed fetuses remains unclear. Due to the fact that DS is a fetal disease with a clear genetic background, and further analyses were conducted combining metabolomics and genomics. The results suggested that metabolite alterations in DS fetuses are partially associated with its genetic etiology; namely, the extra copy of chromosome 21 . The identified pathways for histidine metabolism, neuroactive ligand-receptor interaction, and neural diseases are also located on chromosome 21 (Table III).

Among metabolites that exhibit functional overlap between metabolic alterations and extra copies of chromosome 21 in the DS fetus, it is notable that alterations in glutamine-glutamate metabolism are involved in nearly every 
Table III. Identified common markers in two sets.

\begin{tabular}{|c|c|c|c|c|c|c|c|}
\hline \multirow[b]{2}{*}{ Category } & \multirow[b]{2}{*}{ Metabolite } & \multicolumn{3}{|c|}{ Discovery } & \multicolumn{3}{|c|}{ Validation } \\
\hline & & VIP & $\mathrm{FC}$ & P-value & VIP & $\mathrm{FC}$ & P-value \\
\hline Porphyrin & Coproporphyrin III & 2.8375 & 0.241717 & 0.000683 & 3.26 & 0.270882 & 0.007657 \\
\hline \multirow{2}{*}{ Hormone } & Pregnenolone sulfate & 2.0109 & 0.308604 & 0.001741 & 2.9401 & 0.299152 & 0.000017 \\
\hline & Hydrocortisone (cortisol) & 1.2543 & 1.283218 & 0.085341 & 1.9657 & 1.716536 & 0.002482 \\
\hline \multirow[t]{3}{*}{ Bile acid } & Taurochenodeoxycholate & 2.3718 & 0.465954 & 0.000993 & 3.5658 & 0.388247 & 0.001494 \\
\hline & Glycocholic acid & 1.0539 & 0.58811 & 0.066354 & 1.2245 & 0.613199 & 0.048233 \\
\hline & Taurocholate & 1.0283 & 0.403143 & 0.073469 & 2.1432 & 0.419715 & 0.000974 \\
\hline \multirow[t]{4}{*}{ Amino acid } & L-Arginine & 1.5972 & 0.74232 & 0.029519 & 1.7646 & 0.697481 & 0.031024 \\
\hline & L-Histidine & 1.2185 & 0.73082 & 0.093209 & 1.441 & 0.706818 & 0.041167 \\
\hline & L-Glutamate & 2.2569 & 0.391223 & 0.000213 & 2.4137 & 0.509103 & 0.001985 \\
\hline & L-Glutamine & 1.4845 & 1.885866 & 0.067402 & 1.5919 & 1.58102 & 0.035146 \\
\hline
\end{tabular}

VIP, variable importance in projection; FC, fold change.

Table IV. Common Kyoto Encyclopedia of Genes and Genomes pathways involved with genes in chromosome 21 and amino fluid metabolites from Down syndrome fetuses.

\begin{tabular}{|c|c|c|}
\hline KEGG pathways & Metabolites & Genes in chromosome 21 \\
\hline Galactose metabolism & $\begin{array}{l}\alpha \text {-D-glucose, D-mannose, Myo-inositol, } \\
\text { stachyose }\end{array}$ & PFKL \\
\hline Purine metabolism & L-glutamine, hypoxanthine, adenosime & PDE9A, GART \\
\hline Histidine metabolism & L-histidine, L-methyl-histidine, L-glutamate & FTCD \\
\hline $\mathrm{ABC}$ transporters & $\begin{array}{l}\text { Arginine, histidine, glutamate, glutamine, } \\
\text { mannitol, mannose, myo-inositol }\end{array}$ & ABCG1 \\
\hline $\begin{array}{l}\text { Neuroactive ligand-receptor } \\
\text { interaction }\end{array}$ & $\begin{array}{l}\text { Adenosine, metabotropic glutamate, } \\
\text { dopamine, cortisol, glutamate }\end{array}$ & GIRK1 \\
\hline Parkinson's disease & Dopamine, adenosine & ATP5J, ATP5O, NDUFV3, UBE2G2 \\
\hline Amyotrophic lateral sclerosis & Glutamate, arginine & SOD1 \\
\hline Huntington's disease & Glutamate & ATP5J, ATP5O, NDUFV3, SOD1 \\
\hline
\end{tabular}

PFKL, phosphofructokinase, liver type; PDE9A, Phosphodiesterase 9A; GART, phosphoribosylglycinamide formyltransferase, phosphoribosylglycinamide synthetase, phosphoribosylaminoimidazole synthetase; FTCD, formimidoyltransferase cyclodeaminase; ABCG1, ATP binding cassette subfamily G member 1; GIRK1, glutamate receptor, ionotropic, kainate 1; ATP5J, ATP synthase, $\mathrm{H}^{+}$transporting, mitochondrial Fo complex subunit F6; ATP5O, ATP synthase, $\mathrm{H}^{+}$transporting, mitochondrial $\mathrm{F} 1$ complex, O subunit; NDUFV3, NADH: ubiquinone oxidoreductase subunit V3; UBE2G2, ubiquitin conjugating enzyme E2 G2; SOD1, superoxide dismutase 1, soluble.

overlapping signaling pathway (Table IV). In previous studies of fetal malformations, alterations in glutamine-glutamate have been reported $(13,17,18)$, suggesting a change in a pivotal element of this signaling pathway in fetal development. In DS, aberrations in glutamatergic transmission constituted a major cause of behavioral deficits, and glutamate is an important hippocampal neuron survival factor (36). Clinically, glutamate uptake is significantly decreased in platelets and fibroblasts from DS patients, and a significant deficit in glutamate has been observed in the hippocampus with DS $(37,38)$. In the present study, it was identified that L-glutamate is the most significantly reduced metabolite in fetal AF $(\mathrm{P}<0.005$ for both experimental sets; Table III). Due to the fact that glutamine is synthesized from glutamate and the glutamate-glutamine cycle serves key roles in neuronal activation, the increased glutamine and decreased glutamate may reflect an imbalance in this cycle. Genetic analysis also indicated that several genes identified on chromosome 21 were involved in glutamaterigic transmission. For instance, the mouse Glur-5 gene maps to chromosome 16 (39), and the homologous human GLUR5 gene maps to the corresponding region of human chromosome 21; the dosage imbalance regarding GLUR5 may thereby have a role in the DS phenotype in both mice and humans (39). Therefore, it is hypothesized that the observed 
glutamine-glutamate alterations are tightly associated with the apparent molecular mechanisms of DS etiology.

In conclusion, data from the present study suggest that metabolic identification was variable when taken from different samples in separate experiments. In the DS fetus, alterations in the four metabolic pathways of porphyrin, bile acids, amino acids and hormones were validated, and significant changes in metabolites of coproporphyrin III, pregnenolone sulfate, taurochenodeoxycholate, L-arginine, taurocholate, hydrocortisone, L-histidine, glycocholic acid,L-glutamate and L-glutamine were identified. Analysis of these metabolic alterations associated them with aberrant gene expression on chromosome 21 of DS fetuses, particularly with respect to intellectual impairment and abnormal erythropoiesis. Therefore, alterations in AF metabolites may provide important information in the understanding of fetal disease pathophysiology beyond that of genomics, epigenomics, and proteomics. This may aid in the development of tests for the diagnosis of fetal diseases, providing an additional tool for exploring the etiology of fetal disease.

\section{Acknowledgements}

The authors would like to thank LetPub for its linguistic assistance during the preparation of this manuscript. The present study was supported by the National Key R\&D Program of China (grant no. 2016YFC1000405), the Natural Science Foundation of China (grant no. 81370751) and the Guangdong Natural Science Foundation (grant no. 2014A030313502).

\section{References}

1. Carmichael SL: Birth defects epidemiology. Eur J Med Genet 57: 355-358, 2014.

2. Hui L and Bianchi DW: Cell-free fetal nucleic acids in amniotic fluid. Hum Reprod Update 17: 362-371, 2010.

3. Underwood MA, Gilbert WM and Sherman MP: Amniotic fluid: Not just fetal urine anymore. J Perinatol 25: 341-348, 2005.

4. Da Sacco S, Sedrakyan S, Boldrin F, Giuliani S, Parnigotto P, Habibian R, Warburton D, De Filippo RE and Perin L: Human amniotic fluid as a potential new source of organ specific precursor cells for future regenerative medicine applications. J Urol 183: 1193-1200, 2010.

5. Kamath-Rayne BD, Smith HC, Muglia LJ and Morrow AL: Amniotic fluid: The use of high-dimensional biology to understand fetal well-being. Reprod Sci 21: 6-19, 2013.

6. Larrabee PB, Johnson KL, Lai C, Ordovas J, Cowan JM, Tantravahi U and Bianchi DW: Global gene expression analysis of the living human fetus using cell-free messenger RNA in amniotic fluid. JAMA 293: 836-842, 2005.

7. Hui L, Slonim DK, Wick HC, Johnson KL and Bianchi DW: The amniotic fluid transcriptome: A source of novel information about human fetal development. Obstet Gynecol 119: 111-118, 2011.

8. Queloz PA, Crettaz D, Thadikkaran L, Sapin V, Gallot D, Jani J, Deprest J, Lémery D, Barelli S and Tissot JD: Proteomic analyses of amniotic fluid: Potential applications in health and diseases. J Chromatogr B Analyt Technol Biomed Life Sci 850: 336-342, 2007.

9. Romero R, Kusanovic JP, Gotsch F, Erez O, Vaisbuch E, Mazaki-Tovi S, Moser A, Tam S, Leszyk J, Master SR, et al: Isobaric labeling and tandem mass spectrometry: A novel approach for profiling and quantifying proteins differentially expressed in amniotic fluid in preterm labor with and without intra-amniotic infection/inflammation. J Matern Fetal Neonatal Med 23: 261-280, 2010.

10. Nicholson JK, Connelly J, Lindon JC and Holmes E: Metabonomics: A platform for studying drug toxicity and gene function. Nat Rev Drug Discov 1: 153-161, 2002.
11. Roux A, Lison D, Junot C and Heilier JF: Applications of liquid chromatography coupled to mass spectrometry-based metabolomics in clinical chemistry and toxicology: A review. Clin Biochem 44: 119-135, 2011.

12. Bock JL: Metabolic profiling of amniotic fluid by proton nuclear magnetic resonance spectroscopy: Correlation with fetal maturation and other clinical variables. Clin Chem 40: 56-61, 1994.

13. Graca G, Goodfellow BJ, Barros AS, Diaz S, Duarte IF, Spagou K, Veselkov K, Want EJ, Lindon JC, Carreira IM, et al: UPLC-MS metabolic profiling of second trimester amniotic fluid and maternal urine and comparison with NMR spectral profiling for the identification of pregnancy disorder biomarkers. Mol Biosyst 8: 1243-1254, 2012.

14. Ivanisevic J, Zhu ZJ, Plate L, Tautenhahn R, Chen S, O'Brien PJ, Johnson CH, Marletta MA, Patti GJ and Siuzdak G: Toward 'omic scale metabolite profiling: A dual separation-mass spectrometry approach for coverage of lipid and central carbon metabolism. Anal Chem 85: 6876-6884, 2013.

15. Smith CA, Want EJ, O'Maille G, Abagyan R and Siuzdak G: XCMS: Processing mass spectrometry data for metabolite profiling using nonlinear peak alignment, matching, and identification. Anal Chem 78: 779-787, 2006.

16. Xia J, Sinelnikov IV, Han B and Wishart DS: MetaboAnalyst 3.0-making metabolomics more meaningful. Nucleic Acids Res 43: W251-W257, 2015.

17. Graca G, Duarte IF, Barros AS, Goodfellow BJ, Diaz S, Carreira IM, Couceiro AB, Galhano E and Gil AM: (1)H NMR based metabonomics of human amniotic fluid for the metabolic characterization of fetus malformations. J Proteome Res 8: 4144-4150, 2009.

18. Graca G, Duarte IF, Barros AS, Goodfellow BJ, Diaz SO, Pinto J, Carreira IM, Galhano E, Pita C and Gil AM: Impact of prenatal disorders on the metabolic profile of second trimester amniotic fluid: A nuclear magnetic resonance metabonomic study. J Proteome Res 9: 6016-6024, 2010.

19. Field T and Diego M: Cortisol: The culprit prenatal stress variable. Int J Neurosci 118: 1181, 2008.

20. Feng JH, Yan YE, Liang G, Liu YS, Li XJ, Zhang BJ, Chen LB, $\mathrm{Yu} \mathrm{H}, \mathrm{He} \mathrm{XH}$ and Wang H: Maternal and fetal metabonomic alterations in prenatal nicotine exposure-induced rat intrauterine growth retardation. Mol Cell Endocrinol 394: 59-69, 2014.

21. Marcos J, Craig WY, Palomaki GE, Kloza EM, Haddow JE, Roberson M, Bradley LA and Shackleton $\mathrm{CH}$ : Maternal urine and serum steroid measurements to identify steroid sulfatase deficiency (STSD) in second trimester pregnancies. Prenat Diagn 29: 771-780, 2009.

22. Trivedi DK and Iles RK: Shotgun metabolomic profiles in maternal urine identify potential mass spectral markers of abnormal fetal biochemistry-dihydrouracil and progesterone in the metabolism of Down syndrome. Biomed Chromatogr 29: 1173-1183, 2014.

23. Menon R, Jones J, Gunst PR, Kacerovsky M, Fortunato SJ, Saade GR and Basraon S: Amniotic fluid metabolomic analysis in spontaneous preterm birth. Reprod Sci 21: 791-803, 2014.

24. Herraez E, Lozano E, Poli E, Keitel V, De Luca D, Williamson C, Marin JJ and Macias RI: Role of macrophages in bile acid-induced inflammatory response of fetal lung during maternal cholestasis. J Mol Med (Berl) 92: 359-372, 2013.

25. Kale $\mathrm{E}$ and Kale A: Amniotic fluid amino acid concentrations in fetal skeletal dysplasia. Clin Exp Obstet Gynecol 41: 280-282, 2014.

26. Bloomfield FH, van Zijl PL, Bauer MK and Harding JE: Effects of intrauterine growth restriction and intraamniotic insulin-like growth factor-I treatment on blood and amniotic fluid concentrations and on fetal gut uptake of amino acids in late-gestation ovine fetuses. J Pediatr Gastroenterol Nutr 35: 287-297, 2002.

27. Bednov A, Espinoza J, Betancourt A, Vedernikov Y, Belfort M and Yallampalli C: L-arginine prevents hypoxia-induced vasoconstriction in dual-perfused human placental cotyledons. Placenta 36: 1254-1259, 2015.

28. da Costa CM, de Freitas MR, Brazão V, dos Santos CD, Sala MA, do Prado Júnior JC and Abrahão AA: Does L-arginine availability during the early pregnancy alters the immune response of Trypanosoma cruzi infected and pregnant Wistar rats? Exp Parasitol 142: 59-66, 2014.

29. Pimentel AM, Pereira NR, Costa CA, Mann GE, Cordeiro VS, de Moura RS, Brunini TM, Mendes-Ribeiro AC and Resende AC: L-arginine-nitric oxide pathway and oxidative stress in plasma and platelets of patients with pre-eclampsia. Hypertens Res 36: 783-788, 2013. 
30. Shen SF and Hua CH: Effect of L-arginine on the expression of $\mathrm{Bcl}-2$ and Bax in the placenta of fetal growth restriction. J Matern Fetal Neonatal Med 24: 822-826, 2010.

31. Dudzik D, Revello R, Barbas C and Bartha JL: LC-MS-based metabolomics identification of novel biomarkers of chorioamnionitis and its associated perinatal neurological damage. J Proteome Res 14: 1432-1444, 2015.

32. Sakai T: Biomarkers of lead exposure. Ind Health 38: 127-142, 2000.

33. Chou ST, Byrska-Bishop M, Tober JM, Yao Y, Vandorn D, Opalinska JB, Mills JA, Choi JK, Speck NA, Gadue P, et al: Trisomy 21-associated defects in human primitive hematopoiesis revealed through induced pluripotent stem cells. Proc Natl Acad Sci USA 109: 17573-17578, 2012.

34. Chou ST, Opalinska JB, Yao Y, Fernandes MA, Kalota A, Brooks JS Choi JK, Gewirtz AM, Danet-Desnoyers GA, Nemiroff RL and Weiss MJ: Trisomy 21 enhances human fetal erythro-megakaryocytic development. Blood 112: 4503-4506, 2008.

35. Palmas F, Fattuoni C, Noto A, Barberini L, Dessi A and Fanos V: The choice of amniotic fluid in metabolomics for the monitoring of fetus health. Expert Rev Mol Diagn 16: 473-486, 2016.
36. Bambrick LL, Yarowsky PJ and Krueger BK: Glutamate as a hippocampal neuron survival factor: An inherited defect in the trisomy 16 mouse. Proc Natl Acad Sci USA 92: 9692-9696, 1995.

37. Begni B, Brighina L, Fumagalli L, Andreoni S, Castelli E, Francesconi C, Del Bo R, Bresolin N and Ferrarese C: Altered glutamate uptake in peripheral tissues from Down syndrome patients. Neurosci Lett 343: 73-76, 2003.

38. Reynolds GP and Warner CE: Amino acid neurotransmitter deficits in adult Down's syndrome brain tissue. Neurosci Lett 94: 224-227, 1988.

39. Gregor P, Reeves RH, Jabs EW, Yang X, Dackowski W, Rochelle JM, Brown RH Jr, Haines JL, O'Hara BF, Uhl GR, et al: Chromosomal localization of glutamate receptor genes: Relationship to familial amyotrophic lateral sclerosis and other neurological disorders of mice and humans. Proc Natl Acad Sci USA 90: 3053-3057, 1993. 